\title{
AMENABILITY AND ORLICZ FIGÀ-TALAMANCA HERZ ALGEBRAS
}

\author{
RATTAN LAL and N. SHRAVAN KUMAR \\ (Received 16 October 2019; accepted 11 May 2020; first published online 5 October 2020) \\ Communicated by George Willis
}

\begin{abstract}
In this paper, we characterize amenability of locally compact groups in terms of the properties of Orlicz Figà-Talamanca Herz algebras.
\end{abstract}

2020 Mathematics subject classification: primary 43A07, 43A15; secondary 46J10.

Keywords and phrases: Orlicz space, Orlicz Figà-Talamanca Herz algebra, amenable group, bounded approximate identity, derivation, splittings..

\section{Introduction}

Let $G$ be a locally compact group and let $A_{p}(G)$ be the Figà-Talamanca Herz algebra introduced by Herz [10]. The following theorem on the characterization of amenability in terms of the $A_{p}(G)$ algebras is well known.

THEOREM 1.1. Let $G$ be a locally compact group. Then the following are equivalent:

(a) The group $G$ is amenable.

(b) The Banach algebra $A_{p}(G)$ possesses a bounded approximate identity.

(c) Every closed cofinite ideal of $A_{p}(G)$ is of the form $I(E)$, where $E$ is a finite subset of $G$.

(d) The Banach algebra $A_{p}(G)$ factorizes weakly.

(e) Each homomorphism from $A_{p}(G)$ with finite dimensional range is continuous.

(f) Every derivation of $A_{p}(G)$ into a Banach $A_{p}(G)$-bimodule is continuous.

The equivalence of the statements (a) and (b) was due to Herz [11]. The equivalence of the statements (a), (c), (e) and (f) was due to Forrest [8]. The equivalence of the statements (a) and (d) was due to Losert [15].

The first author would like to thank the University Grants Commission, India, for the research grant. (C) Australian Mathematical Publishing Association Inc. 2020. This is an Open Access article, distributed under the terms of the Creative Commons Attribution licence (http://creativecommons.org/ licenses/by/4.0/), which permits unrestricted re-use, distribution, and reproduction in any medium, provided the original work is properly cited. 
In [16], we have introduced and studied the $L^{\Phi}$-versions of the Figà-Talamanca Herz algebras. Here $L^{\Phi}$ denotes the Orlicz space corresponding to the Young function $\Phi$. The space $A_{\Phi}(G)$ is defined as the space of all continuous functions $u$, where $u$ is of the form

$$
u=\sum_{n=1}^{\infty} f_{n} * \check{g_{n}},
$$

where $f_{n} \in L^{\Phi}(G), g_{n} \in L^{\Psi}(G),(\Phi, \Psi)$ is a pair of complementary Young functions satisfying the $\Delta_{2}$-condition, and

$$
\sum_{n=1}^{\infty} N_{\Phi}\left(f_{n}\right)\left\|g_{n}\right\|_{\psi}<\infty .
$$

This paper has the modest aim of proving the above said equivalent statements in the context of $A_{\Phi}(G)$ algebras. We shall begin with some preliminaries that are needed in the sequel.

\section{Preliminaries}

Let $\Phi: \mathbb{R} \rightarrow[0, \infty]$ be a convex function. Then $\Phi$ is called a Young function if it is symmetric and satisfies $\Phi(0)=0$ and $\lim _{x \rightarrow \infty} \Phi(x)=\infty$. If $\Phi$ is any Young function, then define $\Psi$ as

$$
\Psi(y):=\sup \{x|y|-\Phi(x): x \geq 0\}, y \in \mathbb{R} .
$$

Then $\Psi$ is also a Young function and is termed as the complementary function to $\Phi$. Further, the pair $(\Phi, \Psi)$ is called a complementary pair of Young functions.

Let $G$ be a locally compact group with a left Haar measure $d x$. We say that a Young function $\Phi$ satisfies the $\Delta_{2}$-condition, denoted $\Phi \in \Delta_{2}$, if there exists a constant $K>0$ and $x_{0}>0$ such that $\Phi(2 x) \leq K \Phi(x)$ whenever $x \geq x_{0}$ if $G$ is compact and the same inequality holds with $x_{0}=0$ if $G$ is noncompact. A Young function $\Phi$ is called an $N$-function if it is continuous and satisfies $\Phi(x)=0$ if and only if $x=0$ and $\lim _{x \rightarrow 0} \Phi(x) / x=0, \lim _{x \rightarrow \infty} \Phi(x) / x=\infty$. If $(\Phi, \Psi)$ is a complementary Young pair such that both $\Phi$ and $\Psi$ satisfy the $\Delta_{2}$-condition, then $\Phi$ and $\Psi$ are $N$-functions.

The Orlicz space, denoted $L^{\Phi}(G)$, is a complex vector space consisting of measurable functions, defined as

$$
L^{\Phi}(G)=\left\{f: G \rightarrow \mathbb{C}: \mathrm{f} \text { is measurable and } \int_{G} \Phi(\beta|f|) d x<\infty \text { for some } \beta>0\right\} .
$$

The Orlicz space $L^{\Phi}(G)$ is a Banach space when equipped with the norm

$$
N_{\Phi}(f)=\inf \left\{k>0: \int_{G} \Phi\left(\frac{|f|}{k}\right) d x \leq 1\right\} .
$$

The above norm is called the Luxemburg norm or Gauge norm. If $(\Phi, \Psi)$ is a complementary Young pair, then there is a norm on $L^{\Phi}(G)$, equivalent to the Luxemburg 
norm, given by,

$$
\|f\|_{\Phi}=\sup \left\{\int_{G}|f g| d x: g \in L^{\Psi}(G), \int_{G} \Psi(|g|) d x \leq 1\right\} .
$$

This norm is called the Orlicz norm.

Let $C_{c}(G)$ denote the space of all continuous functions on $G$ with compact support. If a Young function $\Phi$ satisfies the $\Delta_{2}$-condition, then $C_{c}(G)$ is dense in $L^{\Phi}(G)$. Further, if the complementary function $\Psi$ is such that $\Psi$ is continuous and $\Psi(x)=0$ if and only if $x=0$, then the dual of $\left(L^{\Phi}(G), N_{\Phi}(\cdot)\right)$ is isometrically isomorphic to $\left(L^{\Psi}(G),\|\cdot\|_{\Psi}\right)$. In particular, if both $\Phi$ and $\Psi$ satisfy the $\Delta_{2}$-condition, then $L^{\Phi}(G)$ is reflexive.

We say that a Young function $\Phi$ satisfies the Milnes-Akimovič condition (in short, MA condition) if for each $\epsilon>0$ there exists $c_{\epsilon}>1$ and an $x_{1}(\epsilon) \geq 0$ such that

$$
\Phi^{\prime}((1+\epsilon) x) \geq c_{\epsilon} \Phi^{\prime}(x), x \geq x_{1}(\epsilon) .
$$

We would like to mention that the above condition will be used again and again in many of the theorems because of the following result due to Rao [19, Theorem 8].

THEOREM 2.1. Let $G$ be a locally compact group. Then $G$ is amenable if and only iffor each $N$-function $\Phi$ satisfying the $M A$ condition and for each $v \in M_{1}^{+}(G)$, the operator $T_{v}: L^{\Phi} \rightarrow L^{\Phi}$ has norm 1 , where $T_{v}(f)=v * f$.

Since the MA condition will be used again and again, in the form of the Theorem 2.1, we would like to give an example of a Young function which is also an $N$-function and satisfies the MA condition. Consider the Young function $\Phi(x)=e^{x}-x-1,0 \leq x \in \mathbb{R}$. Then $\Phi$ is an $N$-function satisfying the MA condition [21, page 270]. We would also like to give an example of a Young function which is also an $N$-function but does not satisfy the MA condition. Consider the Young function $\Phi(x)=(1+x) \log (1+x)-x, 0 \leq x \in \mathbb{R}$. Then $\Phi$ is an $N$-function which does not satisfy the MA condition [21, page 270].

For more details on Orlicz spaces, we refer the readers to [20].

Let $\Phi$ and $\Psi$ be a pair of complementary Young functions satisfying the $\Delta_{2}$ condition. Let

$$
A_{\Phi}(G)=\left\{u=\sum_{n=1}^{\infty} f_{n} * \check{g_{n}}:\left\{f_{n}\right\} \subset L^{\Phi}(G),\left\{g_{n}\right\} \in L^{\Psi}(G) \text { and } \sum_{n=1}^{\infty} N_{\Phi}\left(f_{n}\right)\left\|g_{n}\right\|_{\Psi}<\infty\right\} .
$$

Note that if $u \in A_{\Phi}(G)$ then $u \in C_{0}(G)$. If $u \in A_{\Phi}(G)$, define $\|u\|_{A_{\Phi}}$ as

$$
\|u\|_{A_{\Phi}}:=\inf \left\{\sum_{n=1}^{\infty} N_{\Phi}\left(f_{n}\right)\left\|g_{n}\right\|_{\Psi}: u=\sum_{n=1}^{\infty} f_{n} * \check{g_{n}}\right\}
$$

The space $A_{\Phi}(G)$ equipped with the above norm and with the pointwise addition and multiplication becomes a commutative Banach algebra [16, Theorem 3.4]. In fact, $A_{\Phi}(G)$ is a commutative, regular and semisimple Banach algebra with spectrum homeomorphic to $G$ [16, Corollary 3.8]. This Banach algebra $A_{\Phi}(G)$ is called the Orlicz Figà-Talamanca Herz algebra. 
Let $\mathcal{B}\left(L^{\Phi}(G)\right)$ be the linear space of all bounded linear operators on $L^{\Phi}(G)$ equipped with the operator norm. For a bounded complex Radon measure $\mu$ on $G$ and $f \in$ $L^{\Phi}(G)$, define $T_{\mu}: L^{\Phi}(G) \rightarrow L^{\Phi}(G)$ by $T_{\mu}(f)=\mu * f$. It is clear that $T_{\mu} \in \mathcal{B}\left(L^{\Phi}(G)\right)$. Let $P M_{\Phi}(G)$ denote the closure of $\left\{T_{\mu}: \mu\right.$ is a bounded complex Radon measure $\}$ in $\mathcal{B}\left(L^{\Phi}(G)\right)$ with respect to the ultraweak topology. Let $C V_{\Phi}(G)$ denote the set of all operators $T: L^{\Phi}(G) \rightarrow L^{\Phi}(G)$ such that $T(f * g)=T(f) * g$ for all $f, g \in C_{c}(G)$. It is clear that $P M_{\Phi}(G) \subset C V_{\Phi}(G)$ and it is proved in [16, Theorem 3.5], that for a locally compact group $G$, the dual of $A_{\Phi}(G)$ is isometrically isomorphic to $P M_{\Psi}(G)$.

Let $\mathcal{A}$ be a regular, semisimple, commutative Banach algebra with the Gelfand structure space $\Delta(\mathcal{A})$. For a closed ideal $I$ of $\mathcal{A}$, the zero set of $I$, denoted by $Z(I)$, is a closed subset of $\Delta(\mathcal{A})$ defined as

$$
Z(I)=\{x \in E: \hat{a}(x)=0 \forall a \in I\} .
$$

For a closed subset $E \subset \Delta(\mathcal{A})$, we define the following ideals in $\mathcal{A}$ :

$$
\begin{aligned}
j_{\mathcal{A}}(E) & =\{a \in \mathcal{A}: \hat{a} \text { has compact support disjoint from } \mathrm{E}\} \\
J_{\mathcal{A}}(E) & =\overline{j_{\mathcal{A}}(E)} \\
I_{\mathcal{A}}(E) & =\{a \in \mathcal{A}: \hat{a}=0 \text { on } E\} .
\end{aligned}
$$

Note that $J_{\mathcal{A}}(E)$ and $I_{\mathcal{A}}(E)$ are closed ideals in $\mathcal{A}$ with their zero set equal to $E$ and $j_{\mathcal{A}}(E) \subseteq I \subseteq I_{\mathcal{A}}(E)$ for any ideal $I$ with zero set $E$. Further, $E$ is said to be a set of spectral synthesis (or a spectral set) for $\mathcal{A}$ if $I_{\mathcal{A}}(E)=J_{\mathcal{A}}(E)$. Let $I_{\mathcal{A}}^{c}(E)$ denote the elements in $I_{\mathcal{A}}(E)$ with compactly supported Gelfand transforms. We say that $E$ is a set of local spectral synthesis if $I_{\mathcal{A}}^{c}(E) \subseteq J_{\mathcal{A}}(E)$. By [16, Theorem 3.6] singletons are sets of spectral synthesis for $A_{\Phi}(G)$. Further, every closed subgroup is a set of local synthesis for $A_{\Phi}(G)$.

The closed set $E$ is a Ditkin set if for every $u \in I_{\mathcal{A}}(E)$, there exists a sequence $\left\{u_{n}\right\} \subset$ $j_{\mathcal{A}}(E)$ such that $u . u_{n}$ converges in norm to $u$; if the condition holds for every compactly supported $u \in I_{\mathcal{A}}(E)$ then $E$ is called a local Ditkin set. If the sequence can be chosen in such a way that it is bounded and is the same for all $u \in I_{\mathcal{A}}(E)$, then we say that $E$ is a strong Ditkin set. Note that every Ditkin set is a set of spectral synthesis. The Banach algebra $\mathcal{A}$ is called a strong Ditkin algebra if all the singletons and the empty set are strong Ditkin sets.

For more on spectral synthesis see [14, 22].

Throughout this paper, $G$ will denote a locally compact group and $(\Phi, \Psi)$ will denote a complementary pair of Young functions satisfying the $\Delta_{2}$-condition.

\section{Amenability and bounded approximate identities}

We begin this section with the main result of this paper on the characterization of amenable groups in terms of the existence of bounded approximate identities in $A_{\Phi}(G)$.

THEOREM 3.1. Let $G$ be a locally compact group and let $\Phi$ satisfy the MA condition. Then $G$ is amenable if and only if $A_{\Phi}(G)$ possesses a bounded approximate identity. 
Proof. Suppose that $G$ is amenable. Let $K$ be a compact subset of $G$ and let $\epsilon>0$. It follows from Leptin's condition [18, Definition 7.1] that there exists a compact set $C$ in $G$ of nonzero measure such that $|K C|<(1+\epsilon)|C|$. Let $u_{K, \epsilon}=(1 /(1+\epsilon)|C|) \chi_{K C} * \check{\chi}_{C}$. Then $u_{K, \epsilon} \in A_{\Phi}(G)$ and

$$
\begin{aligned}
\left\|u_{K, \epsilon}\right\|_{A_{\Phi}} & \leq \frac{1}{(1+\epsilon)|C|} N_{\Phi}\left(\chi_{K C}\right)\left\|\chi_{C}\right\|_{\Psi} \\
& \leq \frac{2}{(1+\epsilon)|C|} N_{\Phi}\left(\chi_{K C}\right) N_{\Psi}\left(\chi_{C}\right) \\
& \leq \frac{2}{(1+\epsilon)|C|}\left[\Phi^{-1}\left(\frac{1}{|K C|}\right)\right]^{-1}\left[\Psi^{-1}\left(\frac{1}{|C|}\right)\right]^{-1} \\
& \leq \frac{2}{(1+\epsilon)|C|}\left[\Phi^{-1}\left(\frac{1}{(1+\epsilon)|C|}\right)\right]^{-1}\left[\Psi^{-1}\left(\frac{1}{(1+\epsilon)|C|}\right)\right]^{-1} \\
& <2 .
\end{aligned}
$$

Consider the set $\Lambda=\{(K, \epsilon): K$ is a compact subset of $G$ and $\epsilon>0\}$ directed as follows: $\left(K_{1}, \epsilon_{1}\right) \prec\left(K_{2}, \epsilon_{2}\right)$ if $K_{1} \subset K_{2}$ and $\epsilon_{2}<\epsilon_{1}$. Now consider the net $\left\{u_{K, \epsilon}\right\}_{(K, \epsilon) \in \Lambda}$ in $A_{\Phi}(G)$. We now claim that $\left\{u_{K, \epsilon}\right\}_{(K, \epsilon) \in \Lambda}$ is an approximate identity for $A_{\Phi}(G)$. Let $f \in$ $A_{\Phi}(G) \cap C_{c}(G)$ be such that supp $f=K$ and let $\epsilon>0$. Then $\left(u_{K, \epsilon} f\right)(x)=f(x) /(1+\epsilon)$ if $x \in K$ and 0 otherwise. Therefore

$$
\left\|u_{K, \epsilon} f-f\right\|_{A_{\Phi}}=\frac{\epsilon}{1+\epsilon}\|f\|_{A_{\Phi}} \leq \epsilon\|f\|_{A_{\Phi}} .
$$

We now proceed further to prove the converse. Suppose that $A_{\Phi}(G)$ posseses an approximate identity $\left\{u_{\alpha}\right\}_{\alpha \in \Lambda}$ bounded by $c$, for some $c>0$. For a positive function $\psi \in C_{c}(G)$, using [16, Theorem 3.5], it can be shown as in [18, Theorem 10.4], that $\|\psi\|_{1}=\left\|T_{\Psi}\right\|_{C V_{\Psi}(G)}$. We now show that a similar equality holds if we replace $\psi$ by a positive measure having compact support. Let $\mu \in M(G)$ be a positive measure having compact support. Choose $f_{0} \in C_{c}(G)$ such that $f_{0}$ is positive, $\breve{f}_{0}=f_{0}$ and $\left\|f_{0}\right\|_{1}=1$. Note that, for every $f \in C_{c}(G)$ with $N_{\Phi}(f) \leq 1$, we have $f_{0} * f$ is also positive and has compact support. Further,

$$
N_{\Phi}\left(f_{0} * f\right) \leq\left\|f_{0}\right\|_{1} N_{\Phi}(f) \leq 1 .
$$

Also, if $f^{\prime} \in C_{c}^{+}(G)$, then

$$
\left\langle f^{\prime} *\left(f_{0} * \check{f}\right), \mu\right\rangle=\left\langle f^{\prime} * \check{f}, \mu * f_{0}\right\rangle .
$$

Thus

$$
\begin{aligned}
\left\|T_{\mu}\right\|_{C V_{\Psi}(G)} & \geq \sup \left\{\left\langle f^{\prime} *\left(f_{0} * \check{f}\right), \mu\right\rangle: f, f^{\prime} \in C_{c}^{+}(G) \text { with } N_{\Phi}(f) \leq 1, N_{\Psi}\left(f^{\prime}\right) \leq 1\right\} \\
& =\sup \left\{\left\langle f^{\prime} * \check{f}, \mu * f_{0}\right\rangle: f, f^{\prime} \in C_{c}^{+}(G) \text { with } N_{\Phi}(f) \leq 1, N_{\Psi}\left(f^{\prime}\right) \leq 1\right\} \\
& =\left\|T_{\mu * f_{0}}\right\|_{C V_{\Psi}(G)}=\left\|\mu * f_{0}\right\|_{1}=\|\mu\|_{M(G)} .
\end{aligned}
$$

As $\left\|T_{\mu}\right\|_{C V_{\Psi}(G)} \leq\|\mu\|_{M(G)}$ for all $\mu \in M(G)$, we have $\left\|T_{\mu}\right\|_{C V_{\Psi}(G)}=\|\mu\|_{M(G)}$ for all positive $\mu$ having compact support. Thus $G$ is amenable, thanks to Theorem 2.1. 
REMARK. Note that the condition that $\Phi$ satisfies the MA condition in the above theorem is needed only while proving the converse, that is, while invoking Theorem 2.1. As mentioned in [19], the assumption that $\Phi$ satisfies the MA condition is needed only to avoid the Riesz-convexity theorem. Note that the proof of the above theorem for the $A_{p}(G)$-algebras uses the Riesz-convexity theorem. Although an extended Riesz-convexity theorem for Orlicz spaces is available, it cannot be used here.

We now begin to prove some corollaries. In the first corollary, we characterize amenability in terms of certain weak*-closed $A_{\Phi}(G)$-submodules of $P M_{\Psi}(G)$.

COROLlARY 3.2. Let $G$ be a locally compact group, $\Phi$ satisfy the MA condition and let $X$ be a weak ${ }^{*}$-closed $A_{\Phi}(G)$-submodule of $P M_{\Psi}(G)$. Then $G$ is amenable if and only if the following statements about $X$ are equivalent:

(a) The space $X$ is invariantly complemented.

(b) The space ${ }^{\perp} X$ has a bounded approximate identity.

PROOF. The proof of the if part follows from Theorem 3.1 and [6, Proposition 6.4]. The only if part follows again from Theorem 3.1 by choosing $X=\{0\}$.

Let $B_{\Phi}(G)=\left\{u \in C(G): u v \in A_{\Phi}(G) \forall v \in A_{\Phi}(G)\right\}$. Then the space $B_{\Phi}(G)$ when equipped with the operator norm becomes a commutative Banach algebra.

COROLlary 3.3. Let $G$ be an amenable group and let $\Phi$ satisfy the MA condition. Then the two norms $\|\cdot\|_{A_{\Phi}(G)}$ and $\|\cdot\|_{B_{\Phi}(G)}$ are equivalent.

Proof. By definition of $B_{\Phi}(G)$, it is clear that, for any $u \in A_{\Phi}(G),\|u\|_{B_{\Phi}(G)} \leq\|u\|_{A_{\Phi}(G)}$. For this inequality, the assumption on the group to be amenable is not needed.

For the other inequality, note that, since $G$ is amenable, by Theorem 3.1, $A_{\Phi}(G)$ possesses a bounded approximate identity $\left\{u_{\alpha}\right\}$ such that $\left\|u_{\alpha}\right\|_{A_{\Phi}(G)} \leq 2 \forall \alpha$. Thus, for any $u \in A_{\Phi}(G)$, we have,

$$
\left\|u_{\alpha} u\right\|_{A_{\Phi}(G)} \leq\left\|u_{\alpha}\right\|_{A_{\Phi}(G)}\|u\|_{B_{\Phi}(G)} \leq 2\|u\|_{B_{\Phi}(G)} .
$$

Hence the result.

One of the classical results of Reiter states that every closed subgroup of a locally compact abelian group is a set of spectral synthesis for the Fourier algebra $A(G)$. This result is known as the subgroup lemma [22]. This result was generalized to locally compact groups by Takesaki and Tatsuuma [23]. For $1<p<\infty$, Herz generalized the subgroup lemma to $A_{p}(G)$ algebras under the assumption that $G$ is amenable. For other generalizations see [2]. Our next corollary is the subgroup lemma for spectral synthesis. The proof of this is an immediate consequence of Theorem 3.1 and [16, Theorem 3.6].

COROLlary 3.4. Let $G$ be an amenable group and let $\Phi$ satisfy the MA condition. Then every closed subgroup is a set of spectral synthesis for $A_{\Phi}(G)$. 


\section{Ideals with bounded approximate identities}

In this section, our aim is to characterize amenable groups in terms of Ditkin sets.

We shall begin this section by introducing some notations. Let $A, B \subset G$ be closed sets of $G$. Let

$$
\begin{aligned}
\mathscr{S}(A, B) & =\left\{u \in B_{\Phi}(G): u(A)=1, u(B)=0\right\}, \\
s(A, B) & = \begin{cases}\inf \left\{\|u\|_{B_{\Phi}(G)}: u \in \mathscr{S}(A, B)\right\} & \text { if } \mathscr{S}(A, B) \neq \emptyset \\
\infty & \text { if } \mathscr{S}(A, B)=\emptyset\end{cases} \\
\mathscr{F}(A) & =\{K \subset G: K \text { is compact, } K \cap A=\emptyset\}, \\
s_{\Phi}(A) & =\sup \{s(A, K): K \in \mathscr{F}(A)\} .
\end{aligned}
$$

Our first result is an analogue of [8, Proposition 3.4]. This theorem proves the existence of bounded approximate identities with some properties, in certain closed ideals.

THEOREM 4.1. Let $G$ be an amenable locally compact group and let $E$ be a closed subset of $G$. If $E$ is a set of synthesis for $A_{\Phi}(G)$ and $s_{\Phi}(E)<\infty$, then the ideal $I(E)$ has a bounded approximate identity $\left\{u_{\alpha}\right\}_{\alpha \in \Lambda}$ such that the following holds:

(a) $\left\|u_{\alpha}\right\|_{A_{\Phi}(G} \leq 8+4 s_{\Phi}(E) \forall \alpha \in \Lambda$;

(b) $u_{\alpha} \in A_{\Phi}(G) \cap C_{c}(G) \forall \alpha \in \Lambda$;

(c) for every compact subset $K$ of $G$ with $K \cap E=\emptyset$, there exists a sequence $\left\{u_{n}\right\}$ from $\left\{u_{\alpha}\right\}$ such that for every $u \in A_{\Phi}(G)$ with $\operatorname{supp}(u) \subset K$, we have $\left\|u u_{n}-u\right\|_{A_{\Phi}(G)} \leq$ $1 / n$.

Proof. Since $G$ is amenable, it follows from the proof of Theorem 3.1, that $A_{\Phi}(G)$ possesses an approximate identity $\left\{u_{K, \epsilon}\right\}_{\mathcal{F}(E) \times \mathbb{R}^{+}}$such that

(i) $\quad\left\|u_{K, \epsilon}\right\| \leq 2$;

(ii) $\operatorname{supp}\left(u_{K}, \epsilon\right)$ is compact; and

(iii) if $v \in A_{\Phi}(G)$ such that $\operatorname{supp}(v) \subseteq K$ then $u_{K, \epsilon} v=v /(1+\epsilon)$.

Since $s_{\Phi}(E)$ is finite, there exist $u_{K} \in \mathscr{S}(E, K)$ such that $\left\|u_{K}\right\|_{B_{\Phi}(G)} \leq s_{\phi}(E)+1$. Let $v_{K, \epsilon}=u_{K, \epsilon}-u_{K, \epsilon} u_{K}$. It is clear that $v_{K, \epsilon} \in I(E)$. This $\left\{v_{K, \epsilon}\right\}_{(K, \epsilon) \in \mathcal{F}(E) \times \mathbb{R}^{+}}$will satisfy the requirements of the theorem.

LEMMA 4.2. Let $K$ be a compact subgroup of a locally compact group $G$. Then $s_{\Phi}(K)$ is finite.

Proof. Let $C$ be a compact subset of $G$ such that $C \cap K=\emptyset$. Choose an open neighbourhood $U$ of $e$ such that $U$ is symmetric, relatively compact and $C^{-1} K \cap$ $K U^{2}=\emptyset$. Let $u(x)=(1 /|K U|) \chi_{K U} * \check{\chi}_{K U}(x)$. Now, it is clear that $u(e)=1,\|u\|_{B_{\Phi}(G)} \leq 1$. Further, note that $u$ is 1 on $K$ and 0 on $C$, that is, $u \in \mathscr{S}(K, C)$. Hence the result.

As an immediate consequence we have the following corollary. 
COROLlary 4.3. Let $G$ be a locally compact amenable group. Then for each $x \in G$, $I(\{x\})$ contains a bounded approximate identity.

PROOF. The proof of this follows from [16, Theorem 3.6], Theorem 4.1 and Lemma 4.2.

Here is the characterization of amenable groups in terms of the Ditkin sets.

COROLlary 4.4. Let $G$ be a locally compact group and let $\Phi$ satisfy the $M A$ condition. Then $G$ is amenable if and only if $A_{\Phi}(G)$ is a strong Ditkin algebra.

PROOF. The proof of this follows from Theorem 3.1 and Corollary 4.3.

\section{Weak factorization and cofinite ideals}

In this section, we characterize amenable groups in terms of weak factorization and cofinite ideals.

For $A_{p}(G)$ algebras, the following theorem was proved by Losert [15].

THEOREM 5.1. Let $G$ be a locally compact group $G$ and let $\Phi$ satisfy the MA condition. Then $G$ is amenable if and only if $A_{\Phi}(G)$ factorizes weakly.

Proof. Let $G$ be amenable. Then the if part follows from Cohen's factorization theorem. We shall now prove the converse. Suppose that $A_{\Phi}(G)$ weakly factorizes. Note that $A_{\Phi}(G)$ is a self-adjoint Banach algebra. Thus, by [9, Theorem 1.3], there exists $c>0$ such that for each compact subset $C$ of $G$ there exists a positive function $u \in A_{\Phi}(G)$ such that $u \geq 1$ on $C$ and $\|u\|_{A_{\Phi}(G)} \leq c$. Observe that, for $\phi \in C_{c}^{+}(G)$, the norm of the convolution operator $\left\|T_{\phi}\right\|_{C V_{\Psi}(G)}$ is equal to the norm of the linear functional

$$
v \mapsto \int v(x) \phi(x) d x
$$

on $A_{\Phi}(G)$. Thus, $\left|\int \phi(x) d x\right| \leq c\left\|T_{\phi}\right\|_{C V_{\Psi}(G)}$, which implies that $\|\phi\|_{1}^{n} \leq c\left\|T_{\phi}\right\|_{C V_{\Psi}(G)}^{n}$ and hence it follows that $\|\phi\|_{1} \leq\left\|T_{\phi}\right\|_{C V_{\Psi}(G)}$. Now proceeding as in the proof of the converse of Theorem 3.1, one can show that $G$ is amenable.

Before we proceed to our next characterization, here are some preparatory lemmas.

LEMMA 5.2. Let $G$ be a amenable group and let $\Phi$ satisfy the MA condition. Then every finite subset is a set of spectral synthesis.

PROOF. The proof of this is an immediate consequence of [12, Theorem 39.24] and Corollary 4.4 .

LEMMA 5.3. Let $G$ be a nonamenable locally compact group and let $I=I(\{e\})$. Then $I^{2}$ is not closed in $A_{\Phi}(G)$, if $\Phi$ satisfies the MA condition.

Proof. Using [16, Theorem 3.6] and Theorem 5.1, the proof of this follows similar lines as in [6, Lemma 5.7].

Our next result is the characterization of amenable groups in terms of cofinite ideals. 
THEOREM 5.4. Let $G$ be a locally compact group and let $\Phi$ satisfy the MA condition. Then the following are equivalent:

(a) $G$ is amenable.

(b) Every cofinite ideal in $A_{\Phi}(G)$ is of the form $I(E)$ for some finite subset $E$ of $G$.

(c) Each homomorphism from $A_{\Phi}(G)$ with finite-dimensional range is continuous.

Proof. (a) $\Rightarrow$ (b). Let $G$ be amenable and let $I$ be a cofinite ideal in $A_{\Phi}(G)$. By [5, Theorem 2.3], it is enough to show that every closed cofinite ideal is idempotent. Therefore, let us assume that $I$ is a closed cofinite ideal in $A_{\Phi}(G)$. Since $I$ is cofinite, the zero set $Z(I)$ is finite and hence, by Lemma 5.2, $Z(I)$ is a set of spectral synthesis. Thus, it follows that $I=I(Z(I))$. Further, by Theorem 4.1, $I(Z(I))$ has a bounded approximate identity and hence it follows from Cohen's factorization theorem that $I$ is idempotent.

(b) $\Rightarrow$ (a) follows from [5, Theorem 2.3] and Lemma 5.3. The equivalence of (b) and (c) follows again from [5, Theorem 2.3].

\section{Derivations and splittings}

In this section, we characterize amenable groups in terms of continuous derivations. Next we study algebraic splittings and strong splittings of the extensions of the algebra $A_{\Phi}(G)$ in the spirit of [17].

We begin this section by showing the existence of a discontinuous derivation. The proof of this Lemma follows from Lemma 5.3 and [5, page 402].

LEMMA 6.1. Let $G$ be a nonamenable group and let $\Phi$ satisfy the MA condition. Then there exists a discontinuous derivation of $A_{\Phi}(G)$ into a finite dimensional commutative Banach $A_{\Phi}(G)$-bimodule.

LEMMA 6.2. Let $G$ be an amenable group and let $I$ be a closed ideal in $A_{\Phi}(G)$ of infinite codimension. Then there exist sequences $\left\{u_{n}\right\},\left\{v_{n}\right\}$ in $A_{\Phi}(G)$ such that $u_{n} v_{1} \cdots v_{n-1} \notin$ I but $u_{n} v_{1} \cdots v_{n-1} u_{1} v_{n} \in$ I for all $n \geq 2$.

Proof. As in the proof of Theorem 5.4, one can show that the zero set $Z(I)$, of an ideal of infinite codimension, is infinite. Now the remaining proof follows exactly as in the proof of [7, Lemma 2].

Here is the characterization of amenable groups in terms of continuous derivations.

THEOREM 6.3. Let $G$ be a locally compact group and let $\Phi$ satisfy the MA condition. Then the following are equivalent:

(a) Every derivation of $A_{\Phi}(G)$ into a Banach $A_{\Phi}(G)$-bimodule is continuous.

(b) Every derivation of $A_{\Phi}(G)$ into a finite-dimensional commutative Banach $A_{\Phi}(G)$-bimodule is continuous.

(c) $G$ is amenable. 
PROOF. (a) $\Rightarrow$ (b) is trivial and (b) $\Rightarrow$ (c) follows from Lemma 6.1. We shall now prove (c) $\Rightarrow$ (a). In order to prove this, it is enough to verify the conditions of [13, Theorem 2] for a closed cofinite ideal of $A_{\Phi}(G)$. But this follows from Theorem 5.4 and Lemma 6.2.

Before we proceed to study algebraic and strong splittings, here are some notations. Let $x, y \in G \cup\{0\}$. For $z \in \mathbb{C}$ and $u \in A_{\Phi}(G)$, define $u . z$ and $z . u$ as follows:

$$
\begin{aligned}
& u . z:=u(x) z \text { for a nonzero } x \text { and } u . z=0 \text { otherwise; } \\
& z . u:=u(y) z \text { for a nonzero } y \text { and } z . u=0 \text { otherwise. }
\end{aligned}
$$

Note that the above left and right action turns $\mathbb{C}$ into an $A_{\Phi}(G)$-bimodule. In order to emphasize the role of $x$ and $y$, we shall denote this bimodule as $\mathbb{C}_{x, y}$.

A linear functional $m$ on $P M_{\Psi}(G)$ is called a mean if $\|m\|=m(I)=1$. A mean $m$ on $P M_{\Psi}(G)$ is said to be topologically invariant if $u . m=u(e) m$ for all $u \in A_{\Phi}(G)$, that is,

$$
\langle T, u . m\rangle=\langle u . T, m\rangle=u(e)\langle T, m\rangle \quad \forall T \in P M_{\Psi}(G), \forall u \in A_{\Phi}(G) .
$$

It is shown in [16, Corollary 6.2] that the set of all topologically invariant means on $P M_{\Psi}(G)$ is nonempty. As a result, by following the arguments given for [17, Lemma 3.1], we have the following Lemma for $A_{\Phi}(G)$.

LEMMA 6.4. If $x \in G$, then there exists an $A_{\Phi}(G)$-bimodule homomorphism $\Theta_{x}$ : $A_{\Phi}(G)^{\prime} \rightarrow \mathbb{C}_{x, x}$ such that $\left\|\Theta_{x}\right\|=\Theta_{x}\left(\delta_{x}\right)=1$.

For algebraic and strong splittings of extensions of Banach algebras, we shall refer to [4].

As a consequence of the above Lemma along with [16, Theorem 3.6] and [16, Corollary 3.8], we have the following Lemma, whose proof is similar to [17, Lemma 3.4].

LEMma 6.5. Let $X$ be a finite-dimensional Banach $A_{\Phi}(G)$-bimodule. Suppose that $X$ is also essential as a left module. Then every singular extension of $A_{\Phi}(G)$ by $X$ splits strongly.

Corollary 6.6. Let $X$ be a finite-dimensional $A_{\Phi}(G)$-bimodule. Then $X$ is isomorphic to $\underset{i=1}{\oplus} \mathbb{C}_{x_{i}, y_{i}}$, for some $n \in \mathbb{N}$ and $x_{i}, y_{i} \in G \cup\{0\}$.

THEOREM 6.7. Let $\Phi$ satisfy the MA condition. If $G$ is amenable, then all finite-dimensional extensions of $A_{\Phi}(G)$ split strongly.

Proof. Let $X$ be a finite-dimensional $A_{\Phi}(G)$-module. As $G$ is amenable, by Theorem 3.1, it follows that $X$ is an essential $A_{\Phi}(G)$-module. Thus, by Lemma 6.5, every singular extension of $A_{\Phi}(G)$ by $X$ splits strongly. Now the conclusion follows from [4, Corollary 1.9.8].

Another proof of this follows from [1, Theorem 4.18], Theorem 4.1 and Theorem 5.4. 
Proposition 6.8. Suppose that $A_{\Phi}(G)$ possesses an approximate identity. Then all the singular finite-dimensional extensions of $A_{\Phi}(G)$ split algebraically.

Proof. Let $X$ be a finite-dimensional $A_{\Phi}(G)$-bimodule. By Corollary 6.6, it follows that

$$
\tilde{H}^{2}\left(A_{\Phi}(G), X\right)=\bigoplus_{i=1}^{n} \tilde{H}^{2}\left(A_{\Phi}(G), \mathbb{C}_{x_{i}, y_{i}}\right),
$$

where $n$ is the dimension of $X$. Note that if $x_{i}$ or $y_{i}$ is nonzero, then by [1, pages 21-22], it follows that $\tilde{H}^{2}\left(A_{\Phi}(G), \mathbb{C}_{x_{i}, y_{i}}\right)=\{0\}$. Further, by [4, Proposition 2.9.34], it follows that $\tilde{H}^{2}\left(A_{\Phi}(G), \mathbb{C}_{0,0}\right)=\{0\}$. Thus the proof follows from [4, Corollary 2.8.13].

\section{Acknowledgement}

The authors would like to thank the unknown referee for carefully reading the manuscript and giving suggestions.

\section{References}

[1] W. G. Bade, H. G. Dales and Z. A. Lykova, Algebraic and Strong Splittings of Extensions of Banach Algebras, Memoirs of the American Mathematical Society, 656 (American Mathematical Society, Providence, RI, 1999).

[2] A. Delaporte and A. Derighetti, 'Invariant projections and convolution operators', Proc. Am. Math. Soc. 129 (2001), 1427-1435.

[3] A. Derighetti, Convolution Operators on Groups, Lecture Notes of the Unione Matematica Italiana, 11 (Springer, Berlin, Heidelberg, 2011).

[4] H. G. Dales, Banach Algebras and Automatic Continuity, London Mathematical Society Monographs (New Series), 24 (Oxford University Press, Oxford, UK, 2000).

[5] H. G. Dales and G. A. Willis, 'Cofinite ideals in Banach algebras, and finite-dimensional representations of group algebras', in: Radical Banach Algebras and Automatic Continuity, Lecture Notes in Mathematics Series, 975 (Springer, Berlin, 1983), 397-407.

[6] B. Forrest, 'Amenability and derivations of the Fourier algebra', Proc. Am. Math. Soc. 104(2) (1988), 437-442.

[7] B. Forrest, 'Amenability and bounded approximate identities in ideals of A(G)', Illinois J. Math. 34(1) (1990), 1-25.

[8] B. Forrest, 'Amenability and the structure of the algebras $A p(G)$ ', Trans. Am. Math. Soc. 343(1) (1994), 233-243.

[9] H. G. Feichtinger, C. G. Graham and E. H. Lakien, 'Nonfactorization in commutative, weakly self-adjoint Banach algebras', Pacific J. Math. 80 (1979), 117-125.

[10] C. Herz, 'The theory of $p$-spaces with an application to convolution operators', Trans. Am. Math. Soc. 154 (1971), 69-82.

[11] C. Herz, 'Harmonic synthesis for subgroups', Ann. Inst. Fourier (Grenoble) 23(3) (1973), 91-123.

[12] E. Hewitt and K. A. Ross, Abstract Harmonic Analysis II (Springer, New York, 1970).

[13] N. P. Jewell, 'Continuity of module of higher derivations', Pacific J. Math. 68 (1977), 91-98.

[14] E. Kaniuth, A Course in Commutative Banach Algebras, Graduate Texts in Mathematics (Springer, New York, 2009).

[15] V. Losert, 'Some properties of groups without the property $\mathrm{P}_{1}$ ', Comment. Math. Helv. 54(1) (1979), 133-139.

[16] R. Lal and N. S. Kumar, 'Orlicz Figà-Talamanca Herz algebras and invariant means', Indag. Math. 30 (2019), 340-354. 
[17] M. S. Monfared, 'Extensions and isomorphisms for the generalized Fourier algebras of a locally compact group', J. Funct. Anal. 198(2) (2003), 413-444.

[18] J. P. Pier, Amenable Locally Compact Groups (Wiley, New York, 1984).

[19] M. M. Rao, 'Convolutions of vector fields-III: amenability and spectral properties', in: Real and Stochastic Analysis (Birkhauser, Boston, MA, 2004), 375-401.

[20] M. M. Rao and Z. D. Ren, Theory of Orlicz Spaces (Dekker, New York, 1991).

[21] M. M. Rao and Z. D. Ren, Applications of Orlicz Spaces (Dekker, New York, 2002).

[22] H. Reiter, Classical Harmonic Analysis and Locally Compact Groups (Oxford University Press, Oxford, UK, 1968).

[23] M. Takesaki and N. Tatsuuma, 'Duality and subgroups II', J. Funct. Anal. 11 (1972), 184-190.

RATTAN LAL, Department of Mathematics,

Indian Institute of Technology Delhi, New Delhi 110016, India

e-mail: rattanlaltank@gmail.com

N. SHRAVAN KUMAR, Department of Mathematics, Indian Institute of Technology Delhi, New Delhi 110016, India

e-mail: shravankumar@maths.iitd.ac.in 